\title{
Numerical simulation of pulsatile blood flow: a study with normal artery, and arteries with single and multiple stenosis
}

\author{
Md. Alamgir Kabir ${ }^{1 *}$, Md. Ferdous Alam² and Md. Ashraf Uddin ${ }^{1}$
}

\author{
* Correspondence: akabir-mat@sust. \\ edu \\ ${ }^{1}$ Department of Mathematics, \\ Shahjalal University of Science and \\ Technology, Sylhet 3114, \\ Bangladesh \\ Full list of author information is \\ available at the end of the article
}

\begin{abstract}
Numerical simulations of pulsatile transitional blood flow through symmetric stenosed arteries with different area reductions were performed to investigate the behavior of the blood. Simulations were carried out through Reynolds averaged Navier-Stokes equations and well-known $k-\omega$ model was used to evaluate the numerical simulations to assess the changes in velocity distribution, pressure drop, and wall shear stress in the stenosed artery, artery with single and double stenosis at different area reduction. This study found a significant difference in stated fluid properties among the three types of arteries. The fluid properties showed a peak in an occurrence at the stenosis for both in the artery with single and double stenosis. The magnitudes of stated fluid properties increase with the increase of the area reduction. Findings may enable risk assessment of patients with cardiovascular diseases and can play a significant role to find a solution to such types of diseases.

Keywords: Pulsatile, Stenosis, Artery, Navier-Stokes, Blood flow
\end{abstract}

\section{Introduction}

For the past few decades, cardiovascular diseases have become the third largest cause of mortality across the globe, where stenoses are of special concern [1, 2]. An abnormal reduction of the cross-sectional area of the artery by deposition of fats and other lipid substances in the beneath of the artery is known as arterial stenosis. Arterial stenosis leads to a significant variation in blood flow parameters, i.e., changes in the velocity gradients and flow structure. In most of the cases, blood flows are considered as laminar [3]. However, it may turn out to turbulent as the intensity of the perturbation of the blood flow created by stenosis. Due to high blood flow velocity at the throat of the stenosis, there may arise high shear stress that causes serious damage to the arterial walls. This affects the behaviors of blood flow [4].

The disease caused by arterial stenosis is known as atherosclerosis [5] which is one of the most widespread diseases of the cardiovascular system globally. The leading causes of death in the world are due to heart diseases such as atherosclerosis [6]. Blood

(c) The Author(s). 2021 Open Access This article is licensed under a Creative Commons Attribution 4.0 International License, which permits use, sharing, adaptation, distribution and reproduction in any medium or format, as long as you give appropriate credit to the original author(s) and the source, provide a link to the Creative Commons licence, and indicate if changes were made. The images or other third party material in this article are included in the article's Creative Commons licence, unless indicated otherwise in a credit line to the material. If material is not included in the article's Creative Commons licence and your intended use is not permitted by statutory regulation or exceeds the permitted use, you will need to obtain permission directly from the copyright holder. To view a copy of this licence, visit http://creativecommons.org/licenses/by/4.0/. The Creative Commons Public Domain Dedication waiver (http://creativecommons.org/publicdomain/zero/1.0/) applies to the data made available in this article, unless otherwise stated in a credit line to the data. 
vessels carry a higher level of cholesterols in the form of low-density lipoprotein (LDL) molecules for a longer period of time [7]. This tends to settle in the innermost layer of the blood vessel which is generally unable to remove sufficient fats from microphage by high-density lipoprotein (HDL). These LDL molecules are generally absorbed by the epithelial cells and form a very thin layer between the tunica interna and tunica muscularis, and this is known as plaque. For a longer period, this plaque spreads along the artery and creates constriction inside the blood vessels which result in a slowdown of the blood flow. These epithelial cells may rapture due to various reasons for example high blood pressure and high wall shear stress (WSS) and expose the fatty core which activates the platelets [8]. This eventually forms a clot inside the vessel [9]. However, sometime this clotting block the entire artery and people may face some physiological disturbance like heart attack and brain strokes which might be the cause of sudden death of a human. Thus, the study on arterial stenosis bears significant importance to understand the details on the behavior of blood flow which causes cardiovascular disease.

Currently, in the era of the vast availability of computational fluid dynamics (CFD) tools and machines with high computational efficiency [10], there is a possibility to perform numerical simulations of the complex physical phenomena occurring inside the human organism, e.g., blood flow in the cardiovascular system [4]. Along with the other fields in fluid dynamics, CFD is already proven to be a prevailing and reliable tool in biomedical applications for the virtual analysis of blood flow patterns [11]. This technique is applied in real-life applications to ensure better product quality and a more robust design. The use of CFD, however, is not only limited to these. In recent times, the application of CFD has grown rapidly in the cardiovascular system studies [12]. For example, CFD is widely used across the spectrum of coronary, valvular, congenital, myocardial, peripheral vascular diseases, risk prediction, and virtual treatment planning [11]. Here in this study to avoid redundant radiation of invasive procedure (i.e., angiography), different non-invasive tools to enhance the understanding of physiological parameters of blood flow were used. Moreover, technological advances in the ultra-sound imaging, the magnetic resonance image, computed tomography, and the computational fluid dynamics are widely used to analyze the effect of the initiation and the progression of the cardiovascular diseases. These are associated with heart failure and brain strokes.

Disturbance of blood flow occurs significantly at low Reynolds number. However, for the comparative of higher severity of constrictions, the flow becomes turbulence which intensifies the viscous and pressure head losses. In the blood flow simulation, a significant number of computational as well as experimental methods are found from the literature which are related to the arterial stenosis [13]. Accuracy of the simulation largely depends on a suitable numerical approach, realistic model geometry, and boundary conditions. Early of the seventieth decay, Lee and Fung [12] carried out a numerical simulation on a locally constricted circular tube to understand atherosclerosis. The flow was considered as steady, homogeneous, and Newtonian viscous. Simulations were restricted for low Reynolds number ranging from 0-25 to avoid instabilities in the numerical procedure that is in for higher Reynolds number in their study. Ahmed and Giddens [7] used Lasser Doppler measurements and flow visualization techniques in a rigid transparent tube with different severity of axisymmetric constrictions of steady. In 
addition, pulsatile flow was taken into consideration to investigate the various effects on flow at the post stenotic zone. This study found that at the early stages of stenosis development, the flow disturbance of discrete oscillation frequency is more important than turbulence. Young and Tsai [14] discussed the detailed description of pressure loss at constraint and separation of flow. The nature of the flow away from occlusion was also explored, i.e., laminar flow, transitional, or turbulent flow. Blood flow due to pumping action of the heart is considered as pulsatile in nature. Ku et al. [9] carried out their experimental study for pulsatile flow. Their study showed correspondence between plaque locations and wall shear stress. The correspondence was shown in such a way that higher shear stress regions are athero-protective and low and oscillating wall shear can lead to various types of plaque formations. Zhang, Bhatti [15] and Mekheimer, Zaher [16] studied entropy analysis on the blood flow through anisotropically tapered arteries filled with magnetic zinc-oxide $(\mathrm{ZnO})$ nanoparticles. Ryval et al. [17] used Wilcox's standard $k-\omega$ model and studied sinusoidal pulsatile flow for the severity of stenosis $75 \%$ and $90 \%$, respectively at Reynolds number 500 and 1000 . In their study, they found that the transitional version of the standard $k-\omega$ model gives a better agreement with the experimental data that of Ahmed and Giddens [7]. Sherwin and Blackburn [13] used both linear stability analyses as well as direct numerical simulation techniques. They considered an axisymmetric pipe with $75 \%$ smooth constrictions and a spectral-element approach with around 20,00,000 nodes to investigate the transition to turbulence of steady and pulsatile flow. Transition to turbulence under pulsatile conditions at a Reynolds number 535 and under steady inlet conditions at Reynolds number 722 were reported in their study.

The blood flow through the artery is spiral. This is due to the heart twisting on its own axis. It is evident from the findings of Stonebridge and Brophy [18] and Stonebridge et al. [19] and Paul et al. [20]. A number of theoretical advantages can be gained when the spiral flow of blood is treated as laminar. For example, reduced turbulence in the artery due to the rotation induced stability and induced lateral force shows a positive effect on arterial damage [19]. A large number of studies conducted to understand the spiral flow of blood in a model of arterial stenosis. However, a complete understanding is still lacking. Many studies found on the effect of both spiral and non-spiral flow in stenosis [21]. Studies on the effect of spiral flow at the different magnitude of spiral speeds are also available in literature. Paul and Lamran [20] showed the spiral flow effect in stenosis at different Reynolds numbers and in their study; they used only two different Reynolds numbers (i.e., 500 and 1000). Kabir et al. [22] used different Reynolds numbers to get an insight into the sensitivity of spiral flow in relation to the Reynolds number. However, blood flow with double stenosis with different area reduction is not well studied yet. Basic knowledge of local hemodynamic parameters in blood flow in the human artery is essential in the diagnosis and treatment of patients with cardiovascular disease [23].

Therefore, the prime objective of this study is to enhance the understanding of pulsatile blood flow along with spiral components by conducting numerical simulations of blood flow with different area reduction. This is done with multiple symmetric arterial stenoses using computational fluid dynamic techniques. Here, the changes of fluid properties in an artery with single stenosis at different area reduction and changes of fluid properties in an artery with double stenosis at different area reduction were studied. Finally, a comparison was performed on blood flow at different stenosis. 


\section{Methods}

\section{Geometry formulation of arteries}

This study aimed to understand the change in velocity distribution, pressure drop, and wall shear stress in the stenosed artery, artery with single and double stenosis at different area reduction. To do this, first, the geometry of three different types of arteries, i.e., normal artery without stenosis, artery with single stenosis and artery with double stenosis (Fig. 1) was made. Later geometry for different area reduction at the stenosis region was made.

The artificial geometrical model of the blood vessel with stenosis was created by using the following formula of cosine curve [7],

$$
\frac{r(Z)}{R}=1-\delta_{c}\left[1+\cos \left(\frac{Z \pi}{D}\right)\right], \quad-D \leq Z \leq D
$$

This equation was modified for double stenosis as follows:

$$
\frac{r(Z)}{R}=\left\{1-\delta_{c}\left[1+\cos \left(\frac{Z \pi}{D}\right)\right], \quad \begin{array}{l}
-D \leq Z \leq D \\
5 D \leq Z \leq 7 D
\end{array}\right.
$$

In eq.1, $r$ and $Z$ are the radial and axial coordinates respectively; $R$ and $D$ are the radius and diameter of the un-stenosed vessel respectively. The percentage of the stenosis was controlled by the parameter $\delta_{\mathrm{c}}$. The constrictions of the artery followed in the cosine curve with the reduction of the area. For single stenosis, the area was reduced as $60 \%$ and $75 \%$. For double stenosis, the area of first and second stenosis was reduced as $60 \%$ and $60 \%, 60 \%$ and $75 \%, 75 \%$ and $60 \%$, and $75 \%$ and $75 \%$. This smooth reduction of the cross-sectional area produced the inside of the vessel by using eq. 1 . This provides a fairly accurate representation of the biological form of arterial stenosis. This was employed previously in theoretical calculations by Deshpande et al. [24] and Din et al. [25]. The entire span of the biological form of the model was taken as $540 \mathrm{~mm}$ (27D) [24], where diameter $D=20 \mathrm{~mm}$ and the length of the upstream, downstream,

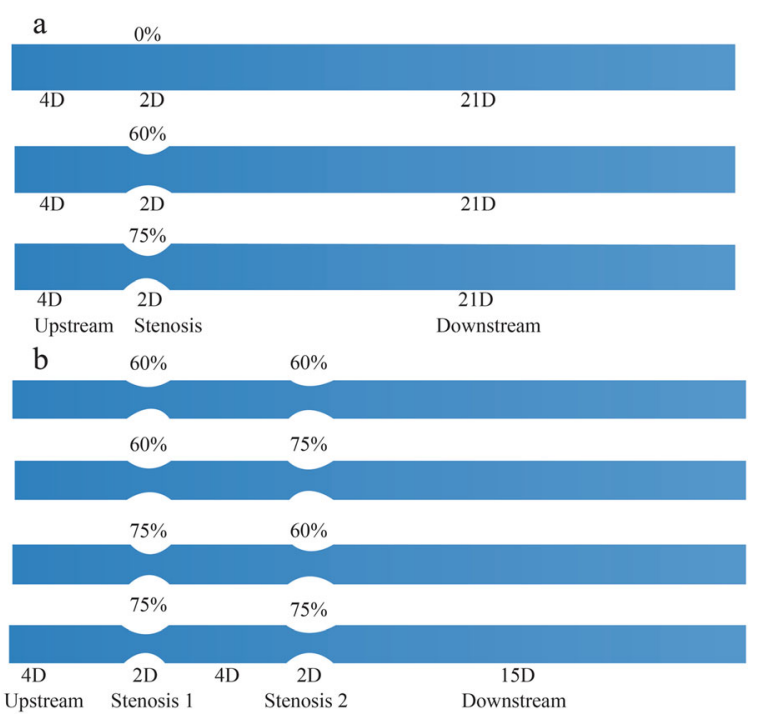

Fig. 1 Model artery without stenosis, with single stenosis (a) and double stenosis (b) with different area reductions for simulations 
and stenosed zone are taken as $4 D, 21 D$, and $2 D$, respectively for single stenosis. $4 \mathrm{D}$, 2D, 4D, 2D, and 15D are the length of upstream, first stenosis, first downstream, second stenosis, and second downstream respectively. In most of the cases, blood flow through different models was considered as incompressible and Newtonianhomogeneous fluid [23] with a density $(\rho)$ of $j 1060 \mathrm{~kg} / \mathrm{m}^{3}$. The constant dynamic viscosity $(\mu)$ was taken as of $3.71 \times 10^{-3} \mathrm{~Pa} \mathrm{~s}$ whereas in this study non-Newtonian model has been used.

The Reynolds averaged Navier-Stokes equations (RANS equations) were considered as the governing equations for blood flow motion. The RANS equations are timeaveraged equations of motion for fluid flow. The equations are generally used to describe the turbulent flows and the idea is to Reynolds decomposition where an instantaneous quantity is decomposed into its time-averaged and fluctuating quantities [26]. RANS models employ an empirical closure hypothesis to compute the components of the Reynolds stress tensor [27]. Classification of RANS models is based on the number of additional differential transport equations required to determine turbulence quantities [28]. After applying the Reynolds time-averaging techniques, the Reynolds averaged Navier-Stokes (RANS) are obtained as tensor form [29] as:

$$
\begin{aligned}
& \frac{\partial \mathrm{u}_{\mathrm{i}}}{\partial \mathrm{x}_{\mathrm{i}}}=0 \\
& \frac{\partial}{\partial t}\left(\rho u_{i}\right)+\frac{\partial}{\partial x_{j}}\left(\rho u_{i} u_{j}\right)=-\frac{\partial p}{\partial x_{i}}+\frac{\partial}{\partial x_{j}}\left[\mu\left(\frac{\partial u_{i}}{\partial x_{j}}+\frac{\partial u_{j}}{\partial x_{i}}\right)\right]+\frac{\partial \tau_{i j}}{\partial x_{j}}
\end{aligned}
$$

In eqs. 2 and $3, x_{i}=(x, y, z)$ are the Cartesian coordinate systems, $u_{i}$ is the mean velocity components, $\rho$ is the density, $p$ is the pressure, and $\tau_{i j}$ are the Reynolds stress (wall shear stress). The Boussinesq hypothesis is employed to model the Reynolds stress $\tau_{i j}$ for our current simulations. The Reynolds stress is the component of the total stress tensor in a fluid obtained from the averaging operation over the Navier-Stokes equations to account for turbulent fluctuations in fluid momentum [30]. Reynolds stress model can successfully capture the turbulence characteristics [31]. Recently, a theoretical basis for determination of the Reynolds stress in canonical flows has been presented [32]. It is based on the turbulence momentum balance for a control volume moving at the local mean flow speed [33]. Therefore, it constitutes a Lagrangian analysis for the momentum transport, which happens to contain the Reynolds stress term [33].

This is given as follows:

$$
\tau_{i j}=-\rho\left\langle u_{i}^{\prime} u_{j}^{\prime}\right\rangle=\mu_{t}\left(\frac{\partial u_{i}}{\partial x_{j}}+\frac{\partial u_{j}}{\partial x_{i}}\right)-\frac{2}{3} \rho k \delta_{i j}
$$

In eq. $4, u_{i}^{\prime}$ are the fluctuating velocity components and $k=\frac{1}{2} \otimes u_{i}^{\prime} u^{\prime}{ }_{j} \bigotimes$ is the turbulent kinetic energy. The turbulent eddy viscosity is denoted as $\mu_{t}$ which is obtained by employing the standard $k-\omega$ model of Wilcox. The eddy viscosity is modeled as:

$$
\mu_{t}=\frac{\rho k}{\omega}
$$

where $\omega$ is the specific dissipation rate. 
The following equation of Wilcox [18] is solved to obtain $k$ and $\omega$ :

$$
\begin{aligned}
& \frac{\partial k}{\partial t}+\frac{\partial k\left\langle u_{j}\right\rangle}{\partial x_{j}}=-\frac{1}{\rho}\left\langle\rho u_{i}^{\prime} u_{j}^{\prime}\right\rangle \frac{\partial\left\langle u_{i}\right\rangle}{\partial x_{j}}-\beta^{*} k \omega+\frac{\partial}{\partial x_{j}}\left[\frac{1}{\rho}\left(\mu+\sigma^{*} \mu_{t}\right) \frac{\partial k}{\partial x_{j}}\right] \\
& \frac{\partial \omega}{\partial t}+\frac{\partial \omega\left\langle u_{j}\right\rangle}{\partial x_{j}}=-\alpha_{1} \frac{\omega}{\rho k}\left\langle\rho u_{i}^{\prime} u_{j}^{\prime}\right\rangle \frac{\partial\left\langle u_{i}\right\rangle}{\partial x_{j}}-\beta \omega^{2}+\frac{\partial}{\partial x_{j}}\left[\frac{1}{\rho}\left(\mu+\sigma \mu_{t}\right) \frac{\partial \omega}{\partial x_{j}}\right]
\end{aligned}
$$

where $\sigma^{*}=0.5, \beta^{*}=0.072, \sigma=0.5, \alpha_{1}=1.0, \beta=0.072$

In computational fluid dynamics, the k-omega $(k-\omega)$ turbulence model is a common two-equation turbulence model, that is used as an approximation for the Reynoldsaveraged Navier-Stokes equations. The model attempts to predict turbulence by two partial differential equations for two variables, $k$ and $\omega$, with the first variable being the turbulence kinetic energy $(k)$ while the second $(\omega)$ is the specific rate of dissipation. Details of the derivation of the model (eqs. 7 and 8) are given in Wilcox [34] and Wilcox [35]. The detailed descriptions of these turbulent models are explained in Varghese et al. [36].

When blood is treated as non-Newtonian fluid and then the viscosity of blood can be calculated from different models such as the Power-law model, Cross model, and Carreau model. In this study, the well-known Carreau model was used with parameters verified by previous studies [20]. The Carreau model is defined as:

$$
\mu(|\dot{\gamma}|)=\mu_{\infty}+\left(\mu_{0}-\mu_{\infty}\right)\left[1+(\lambda \dot{\gamma})^{2}\right]^{(n-1) / 2}
$$

where $\mu_{\infty}(0.00345 \mathrm{~Pa})$ is the infinite shear viscosity, $\mu_{0}(0.056 \mathrm{~Pa})$ is the blood viscosity at zero shear rate, $\gamma$ is the instantaneous shear rate, $\lambda$ (3.313) is the time constant which is associated with the viscosity that changes with shear rate and $n$ is the powerlaw index.

A total of seven inflexible and solid circular model arteries were used as the model artery with different symmetric stenosis. Firstly, the geometry of an inflexible and solid circular model artery without stenosis was developed. Secondly, the geometry of two arteries with single stenosis (i.e., $60 \%$ and $75 \%$ area reduction) was developed. Thirdly, the geometry of four arteries with double stenosis (i.e., 60\% and 60\%, 60\% and 75\%, $75 \%$ and $75 \%$, and $75 \%$ and $60 \%$ area reduction) was developed.

\section{Boundary conditions and computational procedure}

No-slip boundary condition with zero velocity $\left(u_{i}=0\right)$ relative to the boundary along with a pulsatile velocity profile has been imposed at the inlet of the model. The pulsatile velocity profile is computed with the following equation:

$$
V_{\text {inlet }}(t)=\left\{\begin{array}{l}
0.5 \sin [4 \pi(1+0.0160236)]: 0.5 n<t \leq 0.5 n+0.218 \\
0.1: 0.5 n+0.218<t \leq 0.5(n+1)
\end{array}\right.
$$

where $\mathrm{V}(0.5)$ Vis the bulk stream-wise velocity related to the Reynolds number, $\mathrm{Re}=$ $\rho V D / \mu \mathrm{Re}=\rho \mathrm{VD} / \mu$ of the blood flow. Inside the blood-vessel, a proportion of the forward spiral velocity $(\Omega)$ was calculated by using the following equation: 


$$
\Omega=\frac{V}{R} C
$$

A constant $C=1 / 6$ was used to limit the magnitude of the spiral speed.

The outlet of the model has been treated as a pressure outlet and setting for the gauge pressure to become $13332 \mathrm{~Pa}$ as the systolic and diastolic pressure of a healthy human is around $15999 \mathrm{~Pa}$ (i.e., $120 \mathrm{mmHg}$ ) and $10666 \mathrm{~Pa}$ (i.e., $80 \mathrm{mmHg}$ ), respectively. Thus, the average pressure of the two phases, we use $100 \mathrm{mmHg}$ (around 13332 Pascal). All simulations were performed with the commercially available computational fluid dynamics (CFD) software Fluent [37]. This software uses finite volume method for the discretization of the flow governing equations. The finite volume method evaluates partial differential equations in the form of algebraic equations. Pressure based solver was used to solve the flow equations with the implicit formulation method. Besides, the semi-implicit method for pressure-linked equation scheme for pressure-velocity coupling was used. In the spatial discretization process, the least squares based cell scheme was used for the gradient and bounded central differencing scheme was used for momentum. Bounded second-order implicit scheme was used for transient formulation, while the second-order accurate scheme was used for the Poisson-like pressure equation. The minimum time-step size used for the simulation was $1 \times 10^{-2} \mathrm{~s}$ with 10000 numbers of total time-steps. The maximum iterations were 20 per each time step to collect the statistical data. The inlet boundary conditions for the stream-wise velocity was written in C-language using the interface of user defined function of Fluent and linked with the solver. The solution process was initiated using arbitrary values of the velocity components and $k-\omega$, and their residuals are monitored at every iteration. The magnitude of the residuals dropped gradually, which is a strong indicator of stable and accurate solutions. The iteration process was stopped when the residuals are leveled off at $10^{-4}$ and the final converged solutions are achieved.

\section{Results and discussion}

To check the sensitivity of the numerical solutions that are independent of the choice of the grid arrangements, a grid-independent test was carried out. Grid independence test is a mesh convergence test meaning that computing the solution on successively finer grids. The difference between the two refinements is usually taken as a measure of the accuracy of the coarser of the two. It will never show independence unless the difference is of the order of machine zero. We used three different mesh grids points (grids 1,2, and 3) to perform the grid independence tests. The grids are developed automatically using a regular interval using the CFD software. Firstly, the computational domain for grid 1 was discretized into a total number of 77520 control volumes. For grid 2, the total number of control volumes was increased to $32 \%$ of grid 1 (i.e., 102570 control volumes). Finally, for grid 3, total of 127,540 control volumes were used which is approximately $64 \%$ increase of total control volumes of grid 1. Grid independence test suggested that the axial velocity profiles at two different positions (at OD and 1D positions of the model artery with $75 \%$ and $60 \%$ area reduction, respectively) remain similar for the arteries with different control volumes of grids 1, 2, and 3 (Fig. 2a and b). Similar results were found for the centerline axial velocity (Fig. 2c) and the 


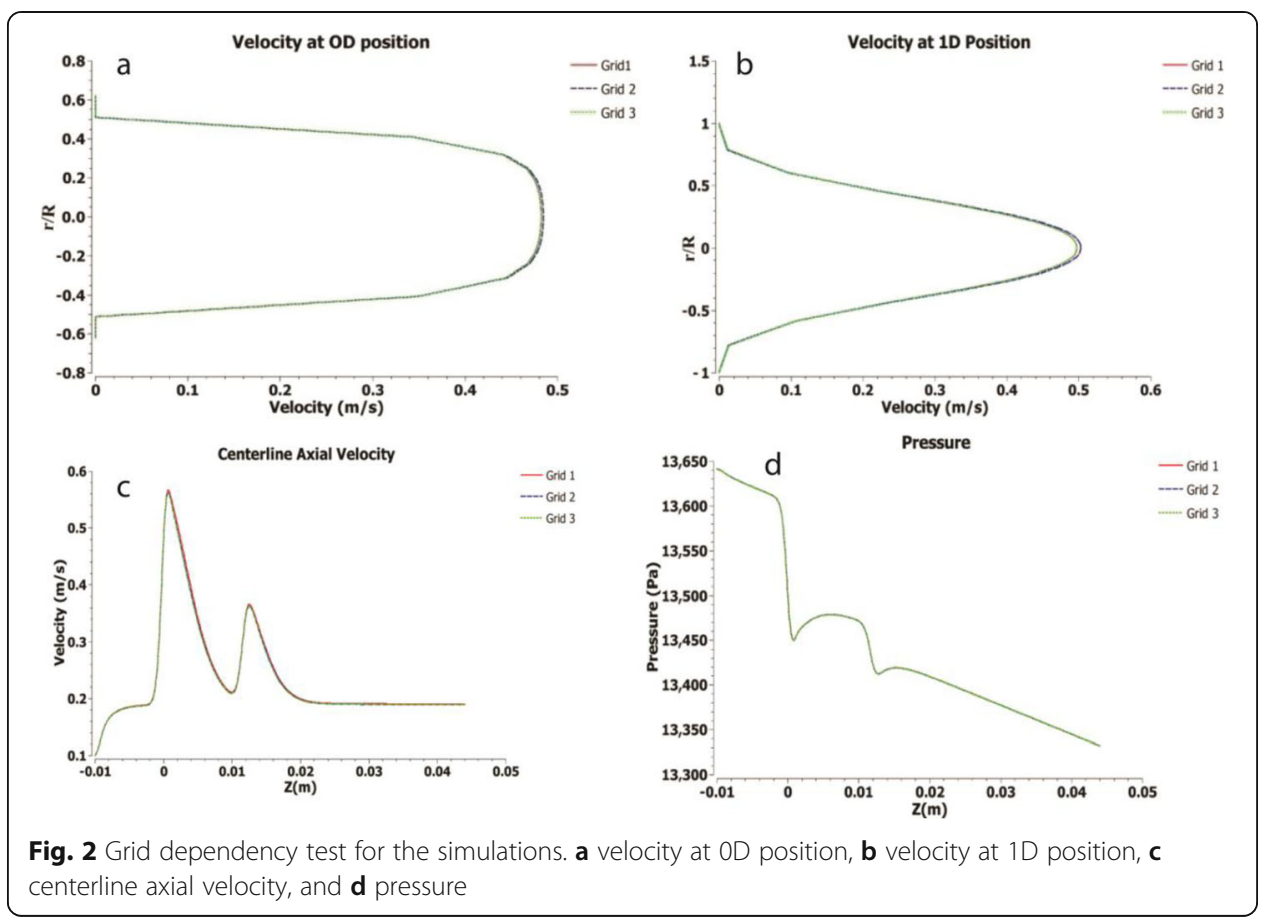

centerline pressure along the flow (Fig. 2d). This suggests that simulations of this study are independent of the grid.

Figure 3 shows the change in axial velocity in normal artery, and artery with single and double stenosis at different area reduction. The axial velocity was almost similar $(0.2 \mathrm{~m} / \mathrm{s})$ throughout the artery while no stenosis was considered. However, when stenosis was considered in the artery, the values drastically change and axial velocity peaks at the stenosis. For single stenosis, one peak in the velocity and for double stenosis two major peaks in the axial velocity was observed. It is notable that axial velocity differs significantly between normal artery and stenosed artery. From the contour plots (Fig. 4a and b), the axial velocity distribution can be observed quite easily. Although the magnitude of axial velocity depends on the percentage of area reduction, for multiple stenoses, the magnitude of the maximum velocity was found almost similar $(0.57 \mathrm{~m} / \mathrm{s})$. Distinctive velocities with rapid change in magnitudes are also found at the throat of the stenosed arteries as well. For both cases, i.e., single and double stenosis, at the stenosis region axial velocity increased with the increase of area reduction. The velocity eventually creates a three dimensional twisting effect on the blood flow. The intensity of the twisting effect increases in the downstream, which can also be explained by the streamlines velocity. Similar results are also reflected in the plot of velocity vectors at different positions (Fig. 5a and b). Our findings of axial velocity are consistent with the study of Mishra and Panda [38], and Young and Tsai [14].

The effect on total pressure in the modeled arteries is shown in Figs. 6 and 7. The pressure distribution in the blood vessel wall is irregular and segmental. In addition, the pressure at the inlet is overall larger than the outlet. Pressure variation is observed with the change in stenosis areas and the larger the stenosis the larger the pressure drop. A maximum pressure drop of $70 \mathrm{~Pa}$ was found in the case of the $75-75 \%$ stenosed 


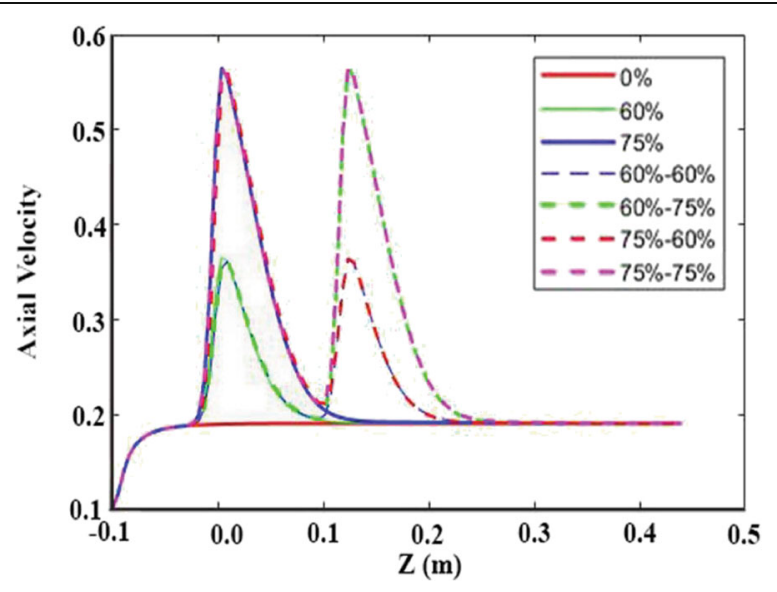

Fig. 3 Changes in axial velocity in normal artery and arteries with single and multiple stenosis

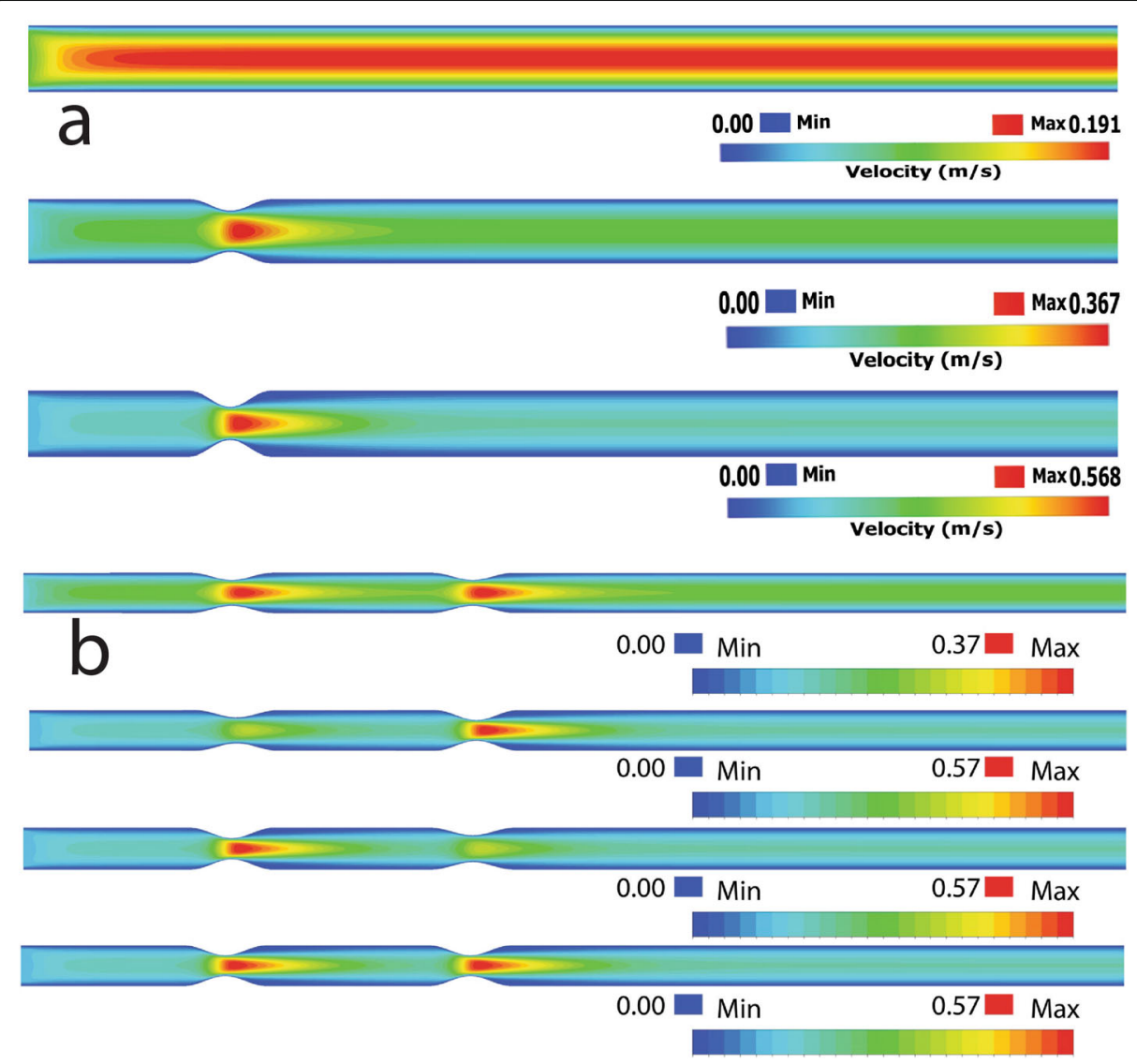

Fig. 4 a Velocity contour for single stenosis along $X Y$ plane, and $\mathbf{b}$ velocity contour for multiple stenoses along XY plane 


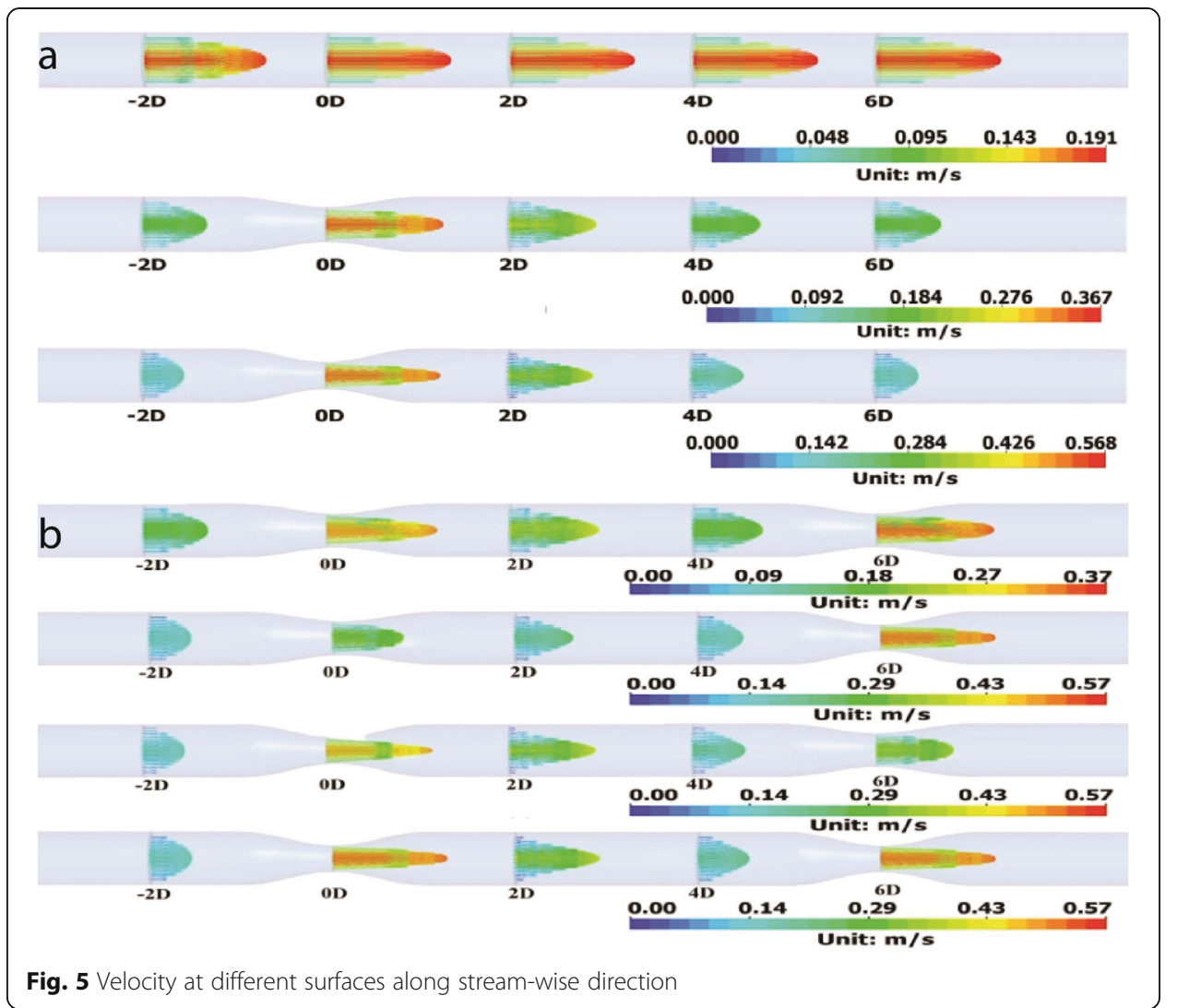

artery. While one pressure drop occurred for single stenosis, multiple pressure drops occurred for the same number of stenosis.

In the artery with single stenosis, pressure peaks before the stenosis and pressure drops largely at the stenosis and remain constant to the rest of the artery. In the artery with single stenosis, pressure peaks before the first stenosis and pressure drops largely after the first stenosis. After this drop, pressure increases again until the start of the second stenosis. Similar to the first stenosis, pressure drops at the second stenosis. The intensity of the pressure increases with the increase in the area reduction on the artery,

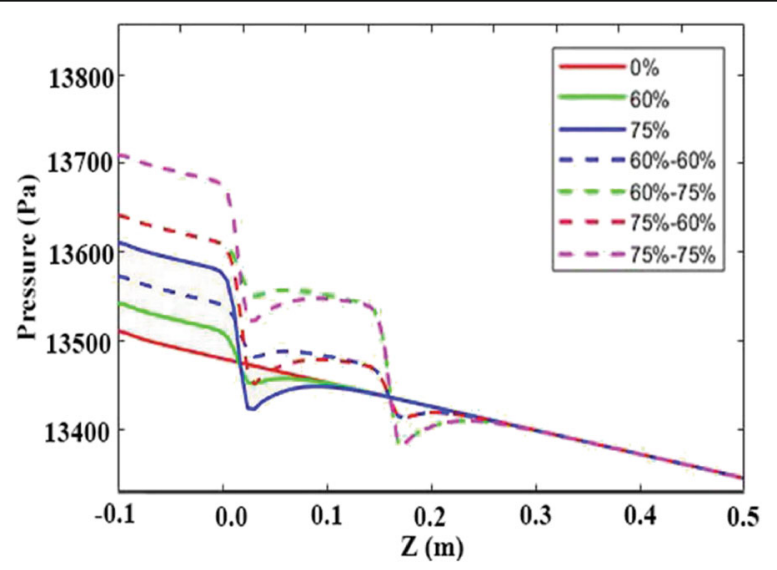

Fig. 6 Variation of pressure in the normal arter and arteries with single and double stenosis 


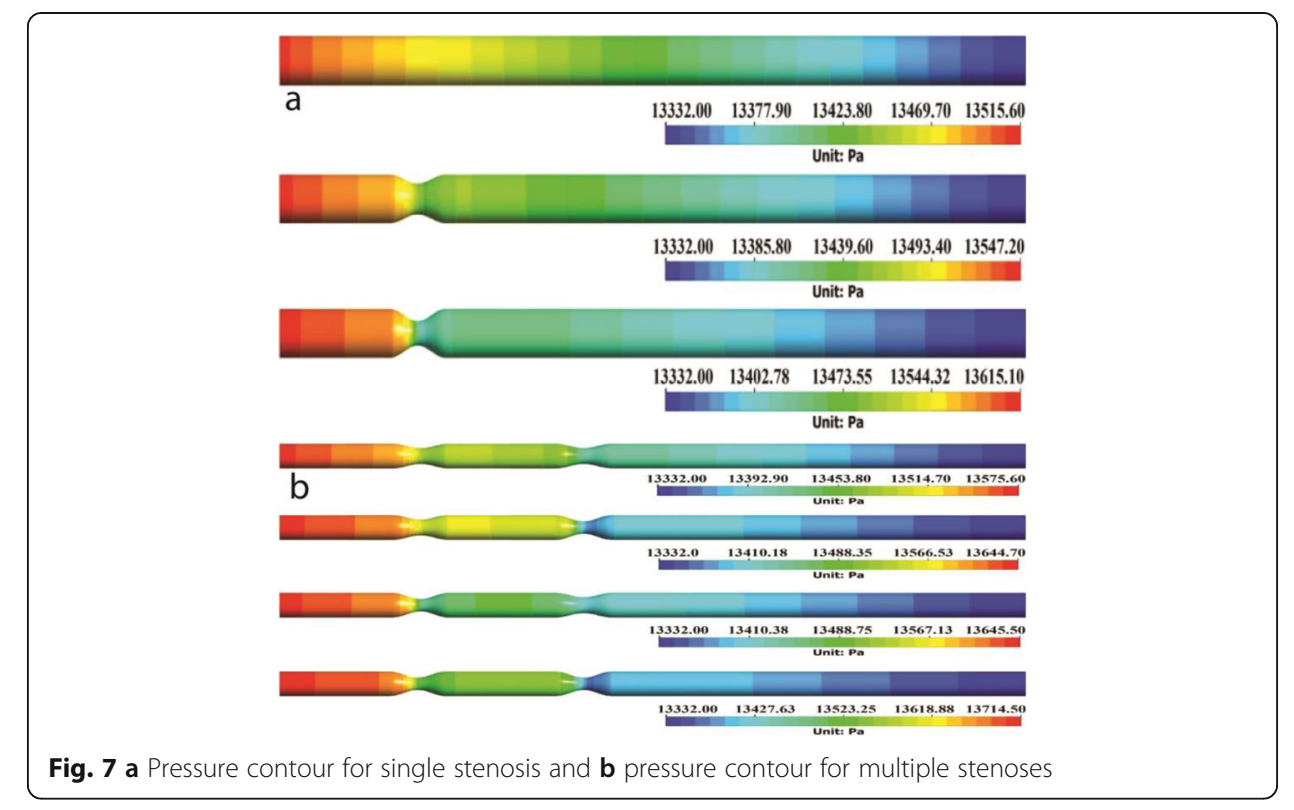

i.e., the higher reduction in area causes a higher level of drops in pressure. The change in pressure after and before the stenosis is also reflected in the pressure contour plot (not shown here). A similar result for the pressure drop at the stenosis was found by Manchester et al. [39] and Abdelsalam, Mekheimer [40]. Venkateswarlu and Rao [41] found that pressure increases with the increase in the area reduction on the artery which is similar to our findings.

The effect on radial velocity in the modeled arteries is shown in Fig. 8. The radial velocity is stable (magnitude is almost zero) throughout the artery without stenosis. However, in the artery with single stenosis, there is a slight peak in the radial velocity at the stenosis. However, this peak is negligible. The radial velocity in the arteries with the double stenosis shows different patterns compared to normal and artery with single stenosis. Radial velocity peaks twice at the artery with double stenosis, i.e., two peaks at the two stenosis regions. It is important to note that the radial velocity is positively related to the area reduction of the artery, and, in the case of multiple stenoses, maximum velocity increase is observed at the first stenosis. Mustapha et al. [42] found two peaks in radial velocity at the two stenosis regions. A positive relation of radial velocity
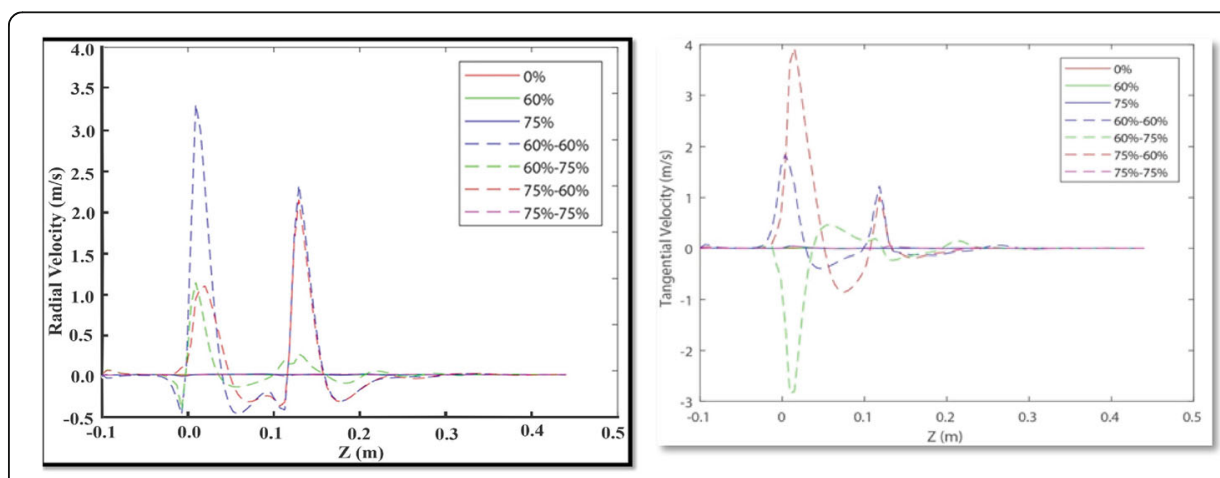

Fig. 8 Effect on radial velocity $\left(\times 10^{-2}\right.$, left $)$ and tangential velocity $\left(\times 10^{-2}\right.$, right $)$ 
with the area reduction of the artery was found by Kanai et al. [43]. These findings are in line with the output of the simulations from our model.

The tangential velocity in the modeled arteries (Fig. 8) shows a similar pattern of radial velocity. The tangential velocity is nearly zero throughout the artery without stenosis, i.e., tangential velocity is stable in the normal artery. In the artery with the single stenosis, there is a slight peak in the tangential velocity at the stenosis and this peak is not significantly high. On the other hand, for multiple stenosed arteries, multiple peaks in the velocity have been observed.

The influence of flow on the wall shear stress (WSS) at different arteries is shown in Fig. 9. WSS plays a very crucial role in the analysis of atherosclerosis in arteries [44]. Even the rupture of plaque can occur due to higher values of WSS. Moreover, low and oscillating wall shear stress is known to be responsible for the formation of plaque [45]. For each artery, the wall shear stress is studied at two perpendicular planes of the artery. The wall shear stress is similar throughout the artery without stenosis. Maximum wall shear stress occurs at upstream to the throat, so this can lead to the possibility of rupture of plaque at this location. This is consistent with the model outputs of Lovett and Rothwell [46] and Sweed and Mekheimer [47]. An increase in severity increases the WSS as flow accelerates more due to an increase in occlusion level to maintain flow rate. The severity of the stenosis increases from 60 to 75\%, WSS increases by almost $117 \%$. Besides, the maximum WSS was found to be 19.07 Pa for $75-60 \%$ stenosis combination. From this value, we can conclude that WSS becomes maximum when the first stenosis has more area reduction than the following stenosis. For double stenosis, the

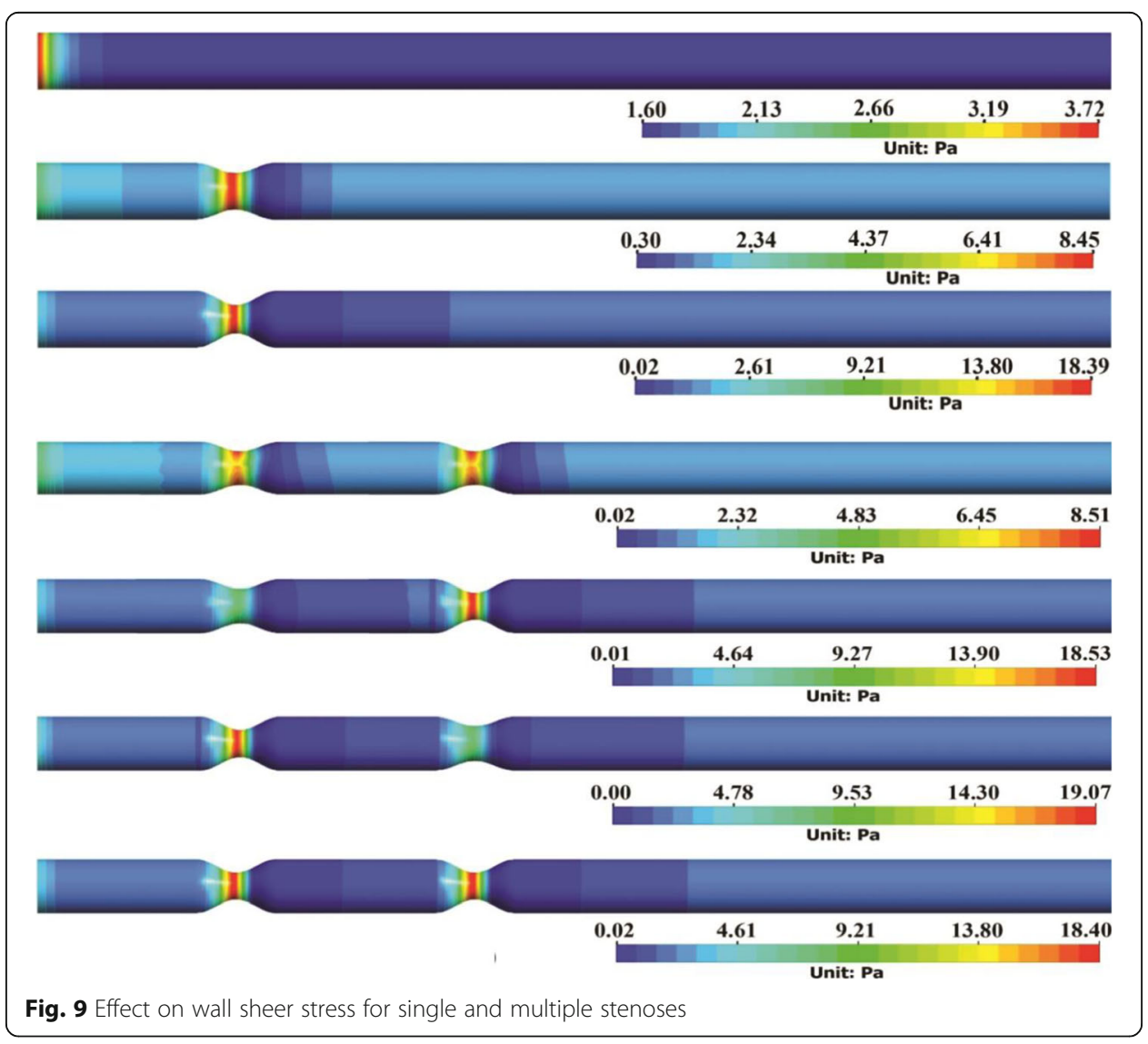


value of WSS increased slightly compared to the single stenosis although the length between the stenosis has been kept constant throughout the experiment. The artery with single stenosis shows one peak while the artery with double stenosis shows double peaks. From our study, it is clear that the WSS increase with the increase in the area of reduction at the stenosis.

The eddy viscosity shows that before the stenosis the eddy viscosity is high. However, after the stenosis, the eddy viscosity drops and remains constant throughout the artery. The turbulence kinetic energy (TKE) also shows that before the stenosis the TKE is higher. However, after the stenosis, the TKE drops and remains constant throughout the artery. In the normal artery (i.e., without any stenosis).

\section{Conclusions}

Arterial stenosis with double stenosis with different area reduction is poorly defined. However, data from existing studies indicate that the arterial stenosis for patients with different area reduction is sufficiently defined. Due to the lack of information on the change in fluid parameters in an artery with double stenosis and different area reduction had created many problems to understand the heart disease problem associated with plaque deposition. This study depicted the changes in velocity distribution, pressure drop, and wall shear stress in the stenosed artery, artery with single and double stenosis at different area reduction. This investigation can play a vital role in the determination of axial velocity, shear stress, and fluid acceleration in particular situations. Since this study has been carried out for a situation, it bears the promise of significant application in cardiovascular disease treatment, which has gained enough popularity. After simulations, we found a significant difference in stated fluid properties among the three types of arteries (i.e., stenosed artery, artery with single and double stenosis at different area reduction). The fluid properties showed a peak in an occurrence at the stenosis for both in the artery with single and double stenosis. Besides, we also found that the magnitudes of stated fluid properties increase with the increase of the area reduction. Our findings may enable risk assessment of patients with cardiovascular diseases and can play a significant role to find a solution to such types of diseases.

\footnotetext{
Abbreviations

$r$ : Radial coordinates; Z: Axial coordinates; $R$ : Radius of the unstenesoed vessel; $D$ : Radius and diameter of the unstenosed vessel; $\delta_{c}$ : Controlling parameters of stenosis; $\mu$ : Viscosity; $x$ : Cartesian coordinate systems; $u_{j}$ : Mean velocity components; $\rho$ : Density; $p$ : Pressure; $\tau_{i j}$ Reynolds stress; $\omega$ : Specific dissipation rate; $k$ : Turbulence kinetic energy; $\gamma$ : Instantaneous shear rate; $\lambda$ : Time constant; v: Bulk stream-wise velocity; Re: Reynolds number; $\Omega$ : Spiral velocity
}

\section{Acknowledgements}

The authors acknowledge the department of mathematics at Shahjalal University of Science and Technology for providing lab facilities for performing simulations.

\section{Authors' contributions}

Md. Almgir Kabir designed the study, did simulations, and wrote the draft version of the manuscript. All other authors contributed equally in updating and revising the manuscript. The author(s) read and approved the final manuscript.

Funding

The study is partially supported by the Ministry of Science and Technology, Government of Bangladesh through NST fellowship (Grant no: F/Y 2018-19 ID 5315). 


\section{Declaration}

\section{Competing interests}

The authors declare that they have no competing interests.

\section{Author details}

${ }^{1}$ Department of Mathematics, Shahjalal University of Science and Technology, Sylhet 3114, Bangladesh. ${ }^{2}$ Department of Mechanical Engineering, Shahjalal University of Science and Technology, Sylhet 3114, Bangladesh.

Received: 23 July 2021 Accepted: 21 September 2021

Published online: 07 November 2021

\section{References}

1. Sulženko J, Pieniazek P (2018) The cardiovascular risk of patients with carotid artery stenosis. Cor et Vasa 60(1):e42-e48. https://doi.org/10.1016/j.crvasa.2017.09.006

2. Bit A, Chattopadhyay H (2014) Three dimensional numerical analysis of hemodynamics of stenosed arteries influencing outlet boundary conditions

3. Virani SS, Alonso A, Benjamin EJ, Bittencourt MS, Callaway CW, Carson AP, Chamberlain AM, Chang AR, Cheng S, Delling FN, Djousse L, Elkind MSV, Ferguson JF, Fornage M, Khan SS, Kissela BM, Knutson KL, Kwan TW, Lackland DT, Lewis TT, Lichtman JH, Longenecker CT, Loop MS, Lutsey PL, Martin SS, Matsushita K, Moran AE, Mussolino ME, Perak AM, Rosamond WD, Roth GA, Sampson UKA, Satou GM, Schroeder EB, Shah SH, Shay CM, Spartano NL, Stokes A, Tirschwell DL, VanWagner L, Tsao CW, American Heart Association Council on Epidemiology and Prevention Statistics Committee and Stroke Statistics Subcommittee (2020) Heart Disease and Stroke Statistics - 2020 update: a report from the American Heart Association. Circulation 141(9):e139-e596. https://doi.org/10.1161/CIR.0000000000000757

4. Tyfa Z, Obidowski D, Reorowicz P, Stefańczyk L, Fortuniak J, Jóźwik K (2018) Numerical simulations of the pulsatile blood flow in the different types of arterial fenestrations: comparable analysis of multiple vascular geometries. Biocybernetics Biomedical Eng 38(2):228-242. https://doi.org/10.1016/j.bbe.2018.01.004

5. Song P, Fang Z, Wang H, Cai Y, Rahimi K, Zhu Y, Fowkes FGR, Fowkes FJ, Rudan I (2020) Global and regional prevalence, burden, and risk factors for carotid atherosclerosis: a systematic review, meta-analysis, and modelling study. Lancet Global Health 8(5):e721-e729. https://doi.org/10.1016/S2214-109X(20)30117-0

6. Roy D, Mazumder O, Sinha A, Khandelwal S (2021) Multimodal cardiovascular model for hemodynamic analysis: simulation study on mitral valve disorders. PLOS ONE 16(3):e0247921. https://doi.org/10.1371/journal.pone.024792

7. Ahmed SA, Giddens DP (1983) Velocity measurements in steady flow through axisymmetric stenoses at moderate Reynolds numbers. J Biomech 16(7):505-516. https://doi.org/10.1016/0021-9290(83)90065-9

8. Bellien J, lacob M, Richard V, Wils J, le Cam-Duchez V, Joannidès R (2021) Evidence for wall shear stress-dependent t-PA release in human conduit arteries: role of endothelial factors and impact of high blood pressure. Hypertens Res 44(3): 310-317. https://doi.org/10.1038/s41440-020-00554-5

9. Ku DN, Giddens DP, Zarins CK, Glagov S (1985) Pulsatile flow and atherosclerosis in the human carotid bifurcation. Positive correlation between plaque location and low oscillating shear stress. Arteriosclerosis 5(3):293-302. https://doi. org/10.1161/01.ATV.5.3.293

10. Zhong L, Zhang JM, Su B, Tan RS, Allen JC, Kassab GS (2018) Application of patient-specific computational fluid dynamics in coronary and intra-cardiac flow simulations: challenges and opportunities. Front Physiol 9(742). https://doi. org/10.3389/fphys.2018.00742

11. Morris PD, Narracott A, von Tengg-Kobligk H, Silva Soto DA, Hsiao S, Lungu A, Evans P, Bressloff NW, Lawford PV, Hose DR, Gunn JP (2016) Computational fluid dynamics modelling in cardiovascular medicine. Heart 102(1):18-28. https://doi. org/10.1136/heartjnl-2015-308044

12. Lee J-S, Fung Y-C (1970) Flow in locally constricted tubes at low Reynolds numbers. J Appl Mechan 37(1):9-16. https:// doi.org/10.1115/1.3408496

13. Sherwin SJ, Blackburn HM (2005) Three-dimensional instabilities and transition of steady and pulsatile axisymmetric stenotic flows. J Fluid Mech 533:297-327. https://doi.org/10.1017/S0022112005004271

14. Young DF, Tsai FY (1973) Flow characteristics in models of arterial stenoses — I. Steady flow. J Biomech 6(4):395-410. https://doi.org/10.1016/0021-9290(73)90099-7

15. Zhang L, Bhatti MM, Marin M, S. Mekheimer K (2020) Entropy analysis on the blood flow through anisotropically tapered arteries filled with magnetic zinc-oxide (ZnO) nanoparticles. Entropy 22(10):1070. https://doi.org/10.3390/e22101070

16. Mekheimer KS, Zaher AZ, Abdellateef Al (2019) Entropy hemodynamics particle-fluid suspension model through eccentric catheterization for time-variant stenotic arterial wall: catheter injection. Int J Geometric Methods Modern Physics 16(11):1950164

17. Ryval J, Straatman AG, Steinman DA (2004) Two-equation turbulence modeling of pulsatile flow in a stenosed tube. J Biomech Eng 126(5):625-635. https://doi.org/10.1115/1.1798055

18. Stonebridge PA, Brophy CM (1991) Spiral laminar flow in arteries? Lancet 338(8779):1360-1361. https://doi.org/10.1016/ 0140-6736(91)92238-W

19. Stonebridge PA, Hoskins PR, Allan PL, Belch JFF (1996) Spiral laminar flow in vivo. Clin Sci (Lond) 91(1):17-21. https://doi. org/10.1042/cs0910017

20. Paul MC, Larman A (2009) Investigation of spiral blood flow in a model of arterial stenosis. Med Eng Phys 31(9):11951203. https://doi.org/10.1016/j.medengphy.2009.07.008

21. Elogail MA, Mekheimer KS (2020) Modulated viscosity-dependent parameters for MHD blood flow in microvessels containing oxytactic microorganisms and nanoparticles. Symmetry 12(12):2114. https://doi.org/10.3390/sym12122114

22. Kabir M, Alam MF, Uddin M (2018) A numerical study on the effects of Reynolds number on blood flow with spiral velocity through regular arterial stenosis. Chiang Mai J Sci 45:2215-2527

23. Buradi A, Mahalingam A (2016) Numerical simulation of pulsatile blood flow in an idealized curved section of a human coronary artery 
24. Deshpande MD, Giddens DP, Mabon RF (1976) Steady laminar flow through modelled vascular stenoses. J Biomech 9(4): 165-174. https://doi.org/10.1016/0021-9290(76)90001-4

25. Ding $\mathrm{G}$ et al (2021) Transitional pulsatile flows with stenosis in a two-dimensional channel. Phys Fluids 33(3):034115

26. Birk L (2019) Reynolds averaged Navier-Stokes equations (RANSE), in Fundamentals of Ship Hydrodynamics, pp 82-93

27. Spalart PR (2000) Strategies for turbulence modelling and simulations. Int J Heat Fluid Flow 21(3):252-263. https://doi. org/10.1016/S0142-727X(00)00007-2

28. Chipongo K, Khiadani M, Sookhak Lari K (2020) Comparison and verification of turbulence Reynolds-averaged NavierStokes closures to model spatially varied flows. Sci Rep 10(1):19059. https://doi.org/10.1038/s41598-020-76128-9

29. Ghalichi F et al (1998) Low Reynolds number turbulence modeling of blood flow in arterial stenoses. Biorheology 35(4): 281-294. https://doi.org/10.1016/S0006-355X(99)80011-0

30. Resende PR, Pinho FT, Cruz DO (2013) A Reynolds stress model for turbulent flows of viscoelastic fluids. J Turbulence 14(12):1-36. https://doi.org/10.1080/14685248.2013.851385

31. Anjum N, Ghani U, Pasha GA, Rashid MU, Latif A, Rana MZY (2018) Reynolds stress modeling of flow characteristics in a vegetated rectangular open channel. Arabian J Sci Eng 43(10):5551-5558. https://doi.org/10.1007/s13369-018-3229-8

32. Lee T-W, Park JE (2017) Integral formula for determination of the Reynolds stress in canonical flow geometries. in Progress in Turbulence VII. Springer International Publishing, Cham

33. Lee TW (2018) The Reynolds stress in turbulence from a Lagrangian perspective. J Phys Commun 2(5):055027. https:// doi.org/10.1088/2399-6528/aac52c

34. Wilcox DC (2008) Formulation of the k-w turbulence model revisited. AIAA J 46(11):2823-2838. https://doi.org/10.2 $514 / 1.36541$

35. Wilcox D (2006) Turbulence modeling for CFD (third edition) (Hardcover)

36. Varghese SS, Frankel SH, Fischer PF (2007) Direct numerical simulation of stenotic flows. Part 1. Steady flow. J Fluid Mechanics 582:253-280. https://doi.org/10.1017/S0022112007005848

37. FLUENT15 (2007) Tutorials guide. Fluent INC, USA

38. Mishra M, Panda P, Tripathy S, Sengupta S, Mishra K (2005) An open randomized comparative study of oral itraconazole pulse and terbinafine pulse in the treatment of onychomycosis. Indian J Dermatol Venereol Leprol 71(4):262-266. https://doi.org/10.4103/0378-6323.16619

39. Manchester EL, Pirola S, Salmasi MY, O'Regan DP, Athanasiou T, Xu XY (2021) Analysis of turbulence effects in a patientspecific aorta with aortic valve stenosis. Cardiovasc Eng Technol 12(4):438-453. https://doi.org/10.1007/s13239-021-0053 6-9

40. Abdelsalam SI, Mekheimer KS, Zaher AZ (2020) Alterations in blood stream by electroosmotic forces of hybrid nanofluid through diseased artery: aneurysmal/stenosed segment. Chin J Phys 67:314-329. https://doi.org/10.1016/j.cjph.2020.07. 011

41. Venkateswarlu K, Rao JA (2004) Numerical solution of unsteady blood flow through an indented tube with atherosclerosis. Indian J Biochem Biophys 41(5):241-245

42. Srivastava VP, Rastogi R (2010) Blood flow through a stenosed catheterized artery: effects of hematocrit and stenosis shape. Comput Math Appl 59(4):1377-1385. https://doi.org/10.1016/j.camwa.2009.12.007

43. Kanai H, lizuka M, Sakamoto K (1970) One of the problems in the measurement of blood pressure by catheter-inserion: wave reflection at the tip of the catheter. Med Biol Eng 8(5):483-496. https://doi.org/10.1007/BF02477185

44. Bajraktari A, Bytyçi I, Henein MY (2020) The relationship between coronary artery wall shear strain and plaque morphology: a systematic review and meta-analysis. Diagnostics (Basel, Switzerland) 10(2):91

45. Alshare A, Tashtoush B (2016) Simulations of magnetohemodynamics in stenosed arteries in diabetic or anemic models. Comput Math Methods Med 2016:8123930

46. Lovett JK, Rothwell PM (2003) Site of carotid plaque ulceration in relation to direction of blood flow: an angiographic and pathological study. Cerebrovasc Dis 16(4):369-375. https://doi.org/10.1159/000072559

47. Sweed NS, Mekheimer KS, el-Kholy A, Abdelwahab AM (2021) Alterations in pulsatile bloodstream with the heat and mass transfer through asymmetric stenosis artery: erythrocytes suspension model. Heat Transfer 50(3):2259-2287. https://doi.org/10.1002/htj.21977

\section{Publisher's Note}

Springer Nature remains neutral with regard to jurisdictional claims in published maps and institutional affiliations.

\section{Submit your manuscript to a SpringerOpen ${ }^{\circ}$ journal and benefit from:}

- Convenient online submission

- Rigorous peer review

- Open access: articles freely available online

- High visibility within the field

- Retaining the copyright to your article

Submit your next manuscript at $\boldsymbol{\nabla}$ springeropen.com 